\title{
Simultaneous Operations in a Two-Dimensional Array of Singlet-Triplet Qubits
}

\author{
Federico Fedele $\odot,{ }^{1, \dagger}$ Anasua Chatterjee $\odot,{ }^{1, \dagger}$ Saeed Fallahi, ${ }^{2,3}$ Geoffrey C. Gardner, ${ }^{3,4}$ \\ Michael J. Manfra, ${ }^{2,3,4,5}$ and Ferdinand Kuemmeth ${ }^{1}{ }^{1, *}$ \\ ${ }^{1}$ Center for Quantum Devices, Niels Bohr Institute, University of Copenhagen, Copenhagen 2100, Denmark \\ ${ }^{2}$ Department of Physics and Astronomy, Purdue University, West Lafayette, Indiana 47907, USA \\ ${ }^{3}$ Birck Nanotechnology Center, Purdue University, West Lafayette, Indiana 47907, USA \\ ${ }^{4}$ Microsoft Quantum Lab Purdue, Purdue University, West Lafayette, Indiana 47907, USA \\ ${ }^{5}$ Schools of Materials Engineering and Electrical and Computer Engineering, Purdue University, West Lafayette, \\ Indiana 47907, USA
}

(Received 5 May 2021; revised 27 July 2021; accepted 30 August 2021; published 8 October 2021)

\begin{abstract}
In many physical approaches to quantum computation, error-correction schemes assume the ability to form two-dimensional qubit arrays with nearest-neighbor couplings and parallel operations at multiple qubit sites. While semiconductor spin qubits exhibit long coherence times relative to their operation speed and single-qubit fidelities above error correction thresholds, multiqubit operations in two-dimensional arrays have been limited by fabrication, operation, and readout challenges. We present a two-by-two array of four singlet-triplet qubits in gallium arsenide and show simultaneous coherent operations and four-qubit measurements via exchange oscillations and frequency-multiplexed single-shot measurements. A larger multielectron quantum dot is fabricated in the center of the array as a tunable interqubit link, which we utilize to demonstrate coherent spin exchange with selected qubits. Our techniques are extensible to other materials, indicating a path towards quantum processors with gate-controlled spin qubits.
\end{abstract}

DOI: 10.1103/PRXQuantum.2.040306

\section{INTRODUCTION}

Semiconducting spin qubits are one of the leading candidates for enabling quantum computation and have demonstrated relatively long coherence times [1,2], figures of merit at the fault tolerance threshold [3], and high-fidelity single- and two-qubit gates [4-7]. Various materials are being investigated, including gallium arsenide (GaAs), silicon, and germanium structures [8]. While linear and twodimensional arrays have been reported up to $9 \times 1$ and $3 \times$ 3 qubit sites [9,10], respectively, most state-of-art GaAs [11-13], silicon [1,14,15] and germanium [16] experiments only performed individual single- and two-qubit operations at a time. (For specific applications in GaAs, however, simultaneous interdot exchange couplings have been demonstrated [13,17-19].) Obstacles for scaling gatecontrolled spin qubit operations to larger arrays include the

\footnotetext{
*kuemmeth@nbi.dk

${ }^{\dagger}$ These authors contributed equally to this work.
}

Published by the American Physical Society under the terms of the Creative Commons Attribution 4.0 International license. Further distribution of this work must maintain attribution to the author(s) and the published article's title, journal citation, and DOI. placement of proximal charge sensors for multiqubit readout, crosstalk between gate electrodes, and reserving space for gate fan-out while preserving nearest-neighbor qubit couplings.

In this work we simultaneously operate and read out four singlet-triplet $\left(S-T_{0}\right)$ qubits, arranged around a central quantum dot in a two-by-two array with four integrated charge sensors. Synchronized voltage pulses applied to eight gate electrodes initialize all qubits and induce coherent Overhauser or exchange rotations. Each fourqubit rotation is followed by spin-to-charge conversion of all qubits, by reading out four local charge sensors via frequency-multiplexed reflectometry. Single-shot measurement outcomes as a function of exchange (dephasing) time clearly reveal simultaneous exchange oscillations (Overhauser rotations) in all four qubits, with quality factors ranging between 3.4 and 5.1, and inhomogeneous dephasing times around $20 \mathrm{~ns}$. By interlacing dephasing and exchange operations, we observe a correlation between low Overhauser gradients and low visibilities of exchange oscillations. The speed of exchange rotations is used to detect a small crosstalk between qubits. By applying voltage pulses to the central multielectron dot, intended as a coupler, we also demonstrate coherent qubit-coupler spin exchange processes. (Qubit-qubit spin exchange, mediated by the coupler, is not demonstrated in this work.) The size 
of the coupler creates sufficient spacing between qubits to facilitate fan-out of gate electrodes and addressability of qubits for individual operation and readout, while still retaining a small overall footprint that makes spin qubits attractive for scaling to large systems.

These techniques constitute a tool set for building quantum processors with programmable connectivities between spin qubits, and may also find use in silicon and germanium quantum circuits.

\section{RESULTS}

All measurements, except where indicated, are performed in a dilution refrigerator at a base temperature of $25 \mathrm{mK}$ in the presence of a static magnetic field of $B_{\|}=120$ $\mathrm{mT}$ applied in the plane of the sample.

\section{A. Device, multiplexed setup, and tuning}

Figure 1(a) shows the two-dimensional layout of the quantum dot circuit, with the four qubits arranged in a $2 \times 2$ array $\left(Q_{1}, Q_{2}, Q_{3}\right.$, and $\left.Q_{4}\right)$. Each qubit is encoded in a double quantum dot (DQD) and operated as a singlettriplet spin qubit [20]. Sensor quantum dots $\left(S_{1-4}\right)$ are placed on the outside of each DQD for single-shot qubit readout via spin-to-charge conversion [21]. For each sensor, this geometry gives good contrast between the charge states within its DQD, while four depletion gates with larger (rectangular) area help screening the electrostatic crosstalk from the other three DQDs.

The gate design also features an elongated gate at the center of the array, connected to circular regions under which the DQDs are formed. This gate is operated in accumulation mode, which not only improves dot confinements and gate control [22], but also accumulates a multielectron dot in the large elongated potential well (white dashed line). This central dot can be pulsed by control voltage $V_{C}$ (intended to mediate coherent spin-exchange between any two qubits) and can also serve as an inner electron reservoir for the DQDs. Fast voltage pulses can also be applied to gates labeled $V_{R i, L i}$ [false colored in gold in Fig. 1(a)], allowing fast manipulation of all DQDs. All pulsed gate electrodes are wirebonded to bias tees located on a high-bandwidth printed-circuit-board sample holder where low-frequency tuning voltages and high-frequency control pulses are combined [23]. On the daughter board of the sample holder, four inductors with different inductances $\left(L_{1}=1200 \mathrm{nH}, L_{2}=560 \mathrm{nH}, L_{3}=750 \mathrm{nH}, L_{4}=\right.$ $910 \mathrm{nH})$ are capacitively coupled to one reflectometry channel of the cryostat, allowing frequency-multiplexed readout of all sensor dots [24].

Figure 1(b) shows charge stability diagrams for all DQDs, measured by digitizing the demodulated reflectometry signal $V_{H i}$ of the proximal sensor dots $S_{i}$ (see below) as a function of the respective plunger gate voltages $V_{R i}$ and $V_{L i}$. All devices are tuned to the $(1,1)$ charge state
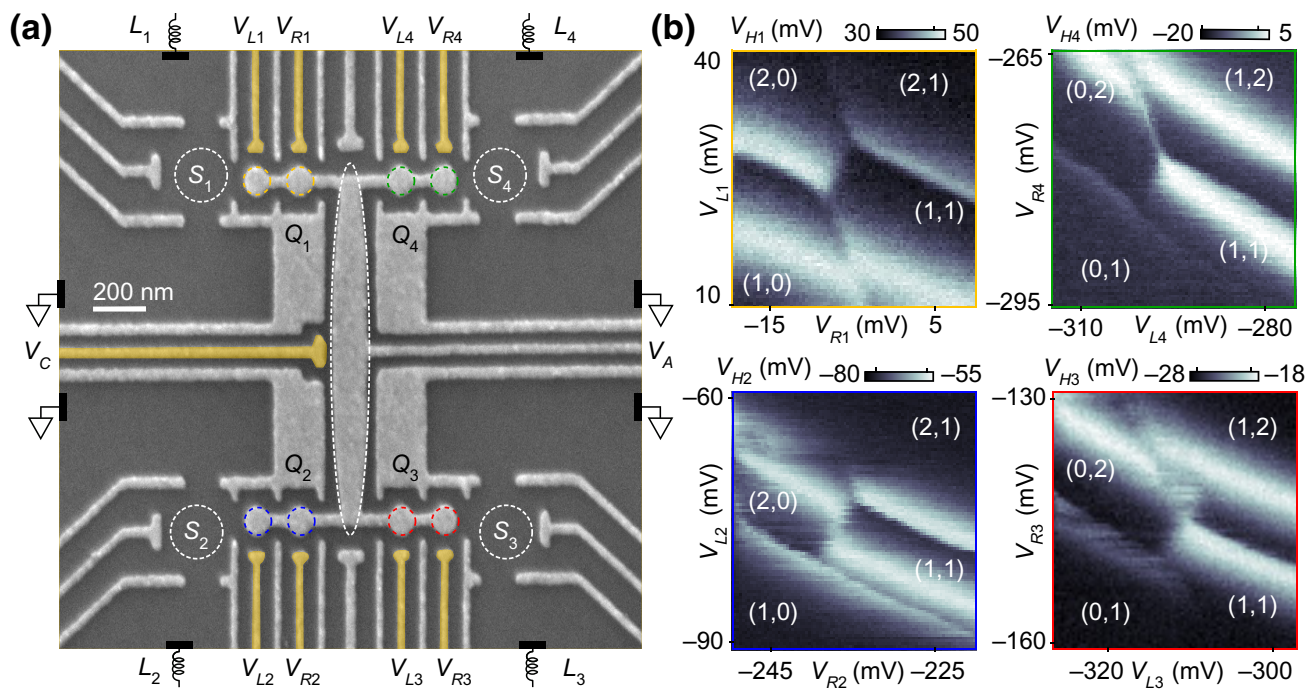

FIG. 1. Two-dimensional array of four tunable GaAs double dots, each implementing one singlet-triplet qubit. (a) Scanning electron micrograph of a device similar to the one measured. All gate electrodes (light gray) are connected to independent tuning voltages ( $V_{i}$ ). Electron reservoirs (black bars) are connected to ohmics, four of which are wirebonded to inductors $L_{1-4}$. Four double dots (small dashed circles) coupled to an elongated multielectron quantum dot (dashed ellipse) are formed by applying a positive voltage to the accumulation gate $\left(V_{A}\right)$. The charge state of each qubit is detected via proximal sensor dots $\left(S_{1-4}\right)$ by applying frequency-multiplexed reflectometry to $L_{1-4}$ and recording the associated homodyne-detected voltages $V_{H 1-H 4}$. Gate electrodes false colored in yellow are connected via bias tees to coaxial lines, allowing the application of fast manipulation pulses $V_{C}(t), V_{L i}(t), V_{R i}(t)$ in Figs. 2-5. (b) Charge stability diagrams of the four singlet-triplet qubits in the absence of pulses. Numbers in parentheses indicate the charge configuration of each double dot. 
with the $(2,0)-(1,1)$ tunnel couplings adjusted to allow coherent operations as singlet-triplet qubits. [Numbers in parentheses $(l, r)$ represent the numbers of electrons on the left and right quantum dots, respectively.]

\section{B. Simultaneous measurements}

The distinct resonance frequencies associated with the inductors allow simultaneous measurements within the array, either by continuous excitation of all inductors [as done in Fig. 1(b)], or by application of short readout bursts within each qubit operation cycle. Each qubit cycle involves a detuning pulse applied to all qubits, taking each DQD from $(2,0)$ to $(1,1)$ and back to $(2,0)$ as illustrated in Fig. 2(a) as a decrease and increase of the detuning parameter $\varepsilon(t)$. For each qubit, comparison of the demodulated reflectometry voltage during the measurement burst $\left(V_{\mathrm{RF}}^{M}\right)$ with the demodulated voltage during the reference burst $\left(V_{\mathrm{RF}}^{R}\right.$, right after initialization of the DQD) allows assignment of single-shot readouts to each qubit, namely singlet if the measurement burst indicates a $(2,0)$ charge state or triplet if the measurement burst indicates a $(1,1)$ charge state.

Each reference and measurement burst applied to the cryostat comprises the four resonance frequencies, yielding qubit-resolved demodulated voltages $V_{H i}$ due to the large separation of resonances in frequency domain (see Sec. IV). Figure 2(b) shows the reflection coefficient of the sample holder [25]. Four well-separated resonances between $130-180 \mathrm{MHz}$ are clearly visible, which disappear one by one if gate voltages associated with the sensor dots are deactivated (dashed line) [24]. (The overall low $S_{11}$ values are caused by intentional attenuation within the cryostat, which exceeds the gain of the cryogenic amplifier in the reflectometry channel.) During an experiment, the reflected bursts are amplified and then demodulated by four parallel homodyne mixers. The resulting four demodulated voltages $V_{H i}$ are measured by a four-channel digitizer, and each channel analyzed to assign single-shot outcomes (Sec. IV). The optimal burst duration depends on the qubit relaxation time and sensor signal-to-noise ratio. With a typical integration time of $10 \mu \mathrm{s}$, we can distinguish $(1,1)$ from $(2,0)$ outcomes with a signal-to-noise ratio of approximately 5 .

Figure 2(c) demonstrates simultaneous four-qubit single-shot readout after each qubit has precessed freely in the Overhauser gradient within its DQD. Each pixel, white for a singlet outcome and black for a triplet outcome, results from application of one eight-dimensional gatevoltage cycle that implements the top cycle in Fig. 2(a) for each qubit by pulsing $V_{L i}$ and $V_{R i}$ [26]. After the qubit is initialized in its $(2,0)$ singlet state $(I)$, a detuning pulse quickly separates the two electrons by pulsing towards $(1,1)$ where spin procession between $|S\rangle$ and $\left|T_{0}\right\rangle$ is driven by the Overhauser field gradient. After precession time $\tau$, the DQD is pulsed back towards $(2,0)$ to implement spinto-charge conversion via Pauli blockade. Repeating this pulse cycle for different values of $\tau$ yields one row for all qubits. A plot of 512 rows obtained right after each other clearly shows four different Overhauser gradients fluctuating over a period of $9 \mathrm{~s}$ at four different locations of the same chip. While no obvious qubit-qubit correlations are visible in these data, an extension of the systematic study in Ref. [26] to four qubits or an implementation of dynamical noise spectroscopy pulses that are specifically designed to measure spatiotemporal qubit-qubit correlations [27] may provide useful insights for optimizing high-fidelity multiqubit operations.

To estimate the inhomogeneous dephasing time $T_{2}^{*}$ for each qubit, we plot at the bottom of Fig. 2(c) the fraction of singlet outcomes, $P_{S}$, for each value of $\tau$. Fitting a semiclassical model of dephasing [20] to the resulting decays yields $T_{2}^{*}$ times of approximately $20 \mathrm{~ns}$, which is typical for singlet-triplet qubits in GaAs.

Figure 2(d) shows coherent exchange oscillations induced simultaneously in all qubits, by plotting $P_{S}$ for the lower pulse cycle in Fig. 2(a). Here, an adiabatic detuning ramp from $(2,0)$ to $(1,1)$ prepares each qubit in the eigenstate of the Overhauser gradient before a quick detuning pulse of duration $\tau$ is applied that turns on an exchange interaction between the two spins. After the exchange pulse, an adiabatic ramp converts the resulting state into either singlet $(2,0)$ or triplet $(1,1)$ charge states. Coherent exchange oscillations in $P_{S}(\tau)$ are clearly observed, with quality factors (3.4-5.1) similar to those observed for nonsymmetric exchange pulses in single-qubit devices [22]. The frequency of exchange oscillations can be tuned individually for each qubit by changing the amplitude of the detuning pulse, and has been chosen for Fig. 2(d) to yield a $\pi$ rotation in $15 \mathrm{~ns}$ for all qubits.

\section{Interleaved operations}

Insight into the finite visibility of exchange oscillations is obtained by alternating qubit exchange cycles and dephasing cycles, as shown in Fig. 3 for two of the qubits. In each alternation cycle, the first measurement burst yields information about exchange rotations, whereas the second burst yields information about the prevailing Overhauser gradient. For convenience, the pulse duration for the exchange pulse is chosen the same as for the dephasing pulse (parameter $\tau$ in the symbolic qubit cycle in the inset of Fig. 3), and repeatedly stepped from 0 to $50 \mathrm{~ns}$ in 60 steps.

Plotting the fraction of singlet outcomes from the first measurement burst again yields conventional exchange oscillations for both qubits [Figs. 3(a) and 3(b)], whereas the second measurement burst yields dephasing data [Figs. 3(c) and 3(d)]. However, if we inspect the underlying single-shot data, as done for $Q_{3}$ in Fig. 3(e), we 
(a)
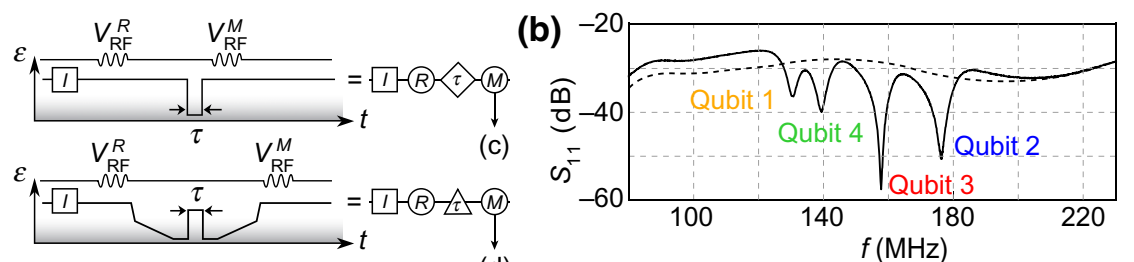

(d)

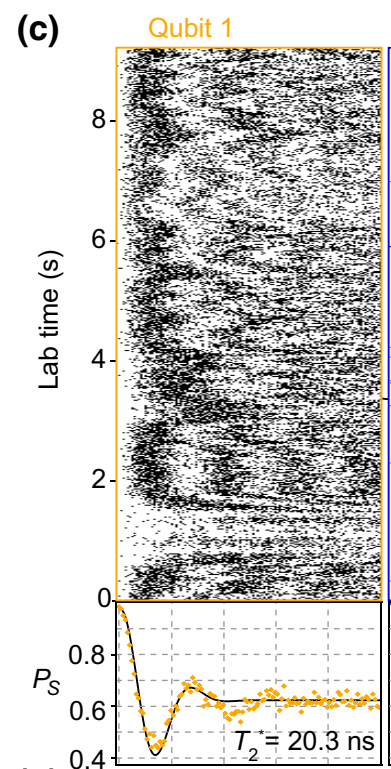

Qubit 2

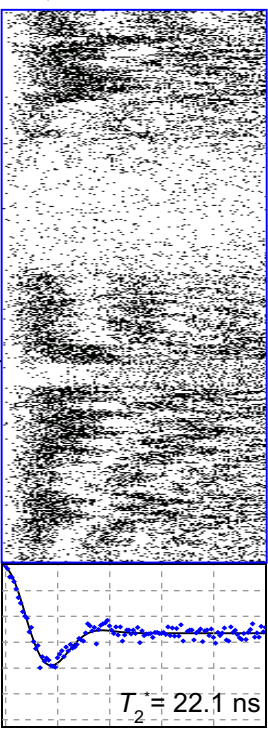

Qubit 3

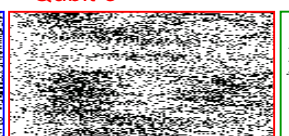

Qubit 4

$\square S \square T$

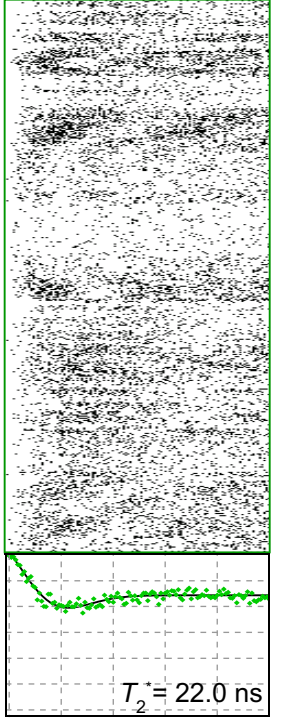

(d)

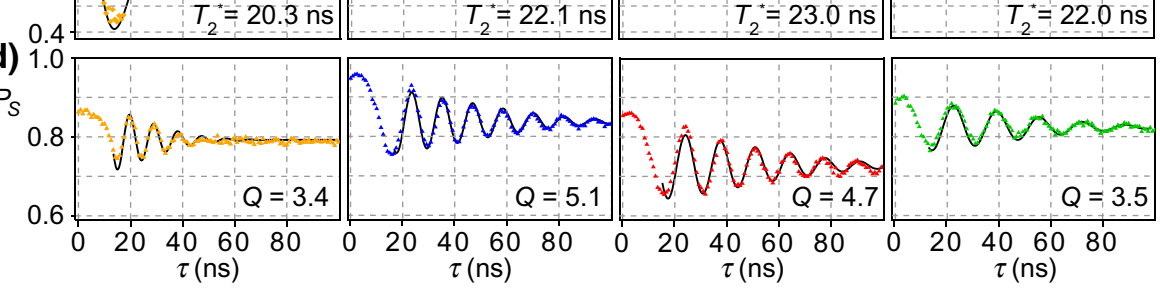

FIG. 2. Simultaneous four-qubit operations. (a) Detuning cycle $\varepsilon(t)$, applied simultaneously to all qubits by appropriate voltage pulses $V_{L i}(t), V_{R i}(t)$, resulting in dephasing operations (top) or exchange rotations (bottom). Qubit initialization (I) is followed by a reference measurement $(R)$ of the reflectometry signal $\left(V_{\mathrm{RF}}^{R}\right)$, a dephasing or exchange pulse of duration $\tau$, and a measurement $(M)$ of the reflectometry signal $\left(V_{\mathrm{RF}}^{M}\right)$ immediately after the final rise of $\varepsilon(t)$ (spin-to-charge conversion). (b) Reflection coefficient of the reflectometry setup as a function of the carrier frequency, in the presence (black line) and absence (dashed line) of tuning voltages applied to all gate electrodes. Four distinct resonances, arising from different inductance values used for $L_{1-4}$, allow frequencymultiplexed single-shot readout of all four qubits. (c) Single-shot outcomes of 61440 dephasing cycles applied simultaneously to all four qubits. Each singlet outcome is represented by one white pixel. Each row represents 120 cycles with increasing values for $\tau$. Acquisition time for 512 rows is $9 \mathrm{~s}$. Bottom panels show the fraction of singlet outcomes, $P_{S}$, obtained by averaging the top panels along the laboratory time. Fits to the data (solid lines) yield $T_{2}^{*}$ times similar for all four qubits. (d) Fraction of singlet outcomes, $P_{S}(\tau)$, for 61440 exchange-rotation cycles applied simultaneously to all four qubits.

observe a temporal correlation between periods of low visibility of exchange oscillations and low Overhauser gradients. We speculate that this indicates a failure of adiabatic conversions between $(0,2)$ singlet states and $(1,1)$ nuclear eigenstates $(|\uparrow \downarrow\rangle$ or $|\downarrow \uparrow\rangle)$ in periods of low Overhauser gradients, which could be mitigated by lowering the detuning ramp rate before and after the exchange pulse [cf. Fig. 2(a)]. A similar visibility reduction for exchange oscillations can occur during periods of large Overhauser gradients, via a mechanism that reduces the triplet lifetime in the measurement configuration of the qubit's DQD [28].
More generally, knowledge of slowly fluctuating parameters in a multiqubit processor, obtained from different types of interlaced diagnostic qubit operation cycles, may prove helpful for near-term applications of small and noisy quantum circuits.

\section{Crosstalk between qubits}

Fine tuning and optimization of simultaneous qubit operations may require detecting and accounting for capacitive crosstalk within qubit arrays. We test for qubitqubit crosstalk caused by simultaneous exchange pulses 

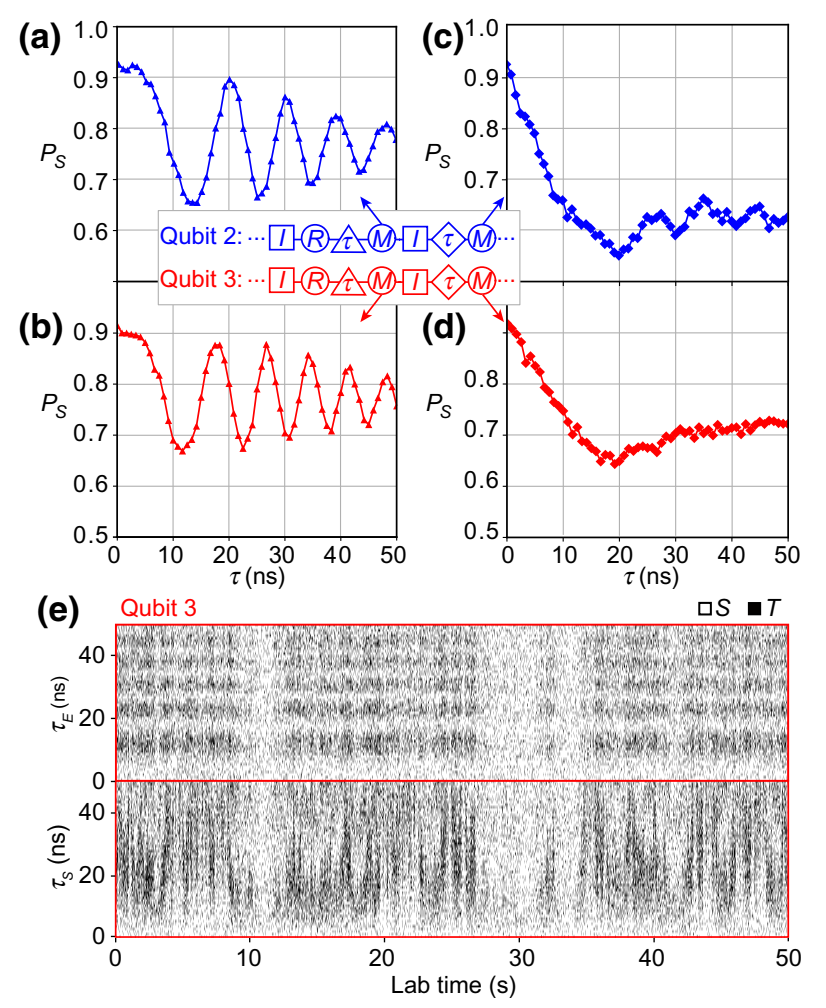

FIG. 3. Interleaved qubit operations applied to $Q_{2}$ and $Q_{3}$. Simultaneous qubit cycles are symbolically represented as in Fig. 2(a). (a)-(d) Fraction of singlet outcomes, $P_{S}(\tau)$, when repeatedly applying an alternating sequence of dephasing and exchange-rotation cycles to both qubits. The value of $\tau$ is changed after each alternation. Measurement outcomes following the exchange pulse [dephasing pulse] are represented in panels (a) and (b) [panels (c) and (d)]. (e) Nonaveraged representation of all single-shot outcomes associated with panels (b) and (d), plotted as a function of $\tau$ for the exchange pulse $\left(\tau_{E}\right)$ or dephasing pulse $\left(\tau_{S}\right)$ and laboratory time. Each singlet outcome for $Q_{3}$ is represented by one pixel. Periods of low Overhauser gradients correlate with periods of low-visibility exchange rotations.

by modifying the interleaved operations of Fig. 3 such that the exchange coupling strength within one qubit $\left(Q_{3}\right)$ can be measured in the presence and absence of concurrent exchange pulses applied to its neighbor qubit $\left(Q_{2}\right)$. Figure 4(a) shows the specific waveforms used for the measurements in Fig. 4(b), with interaction times $\tau_{E}=$ $0, \ldots, 50 \mathrm{~ns}$ varied equally for both qubits in the presence and absence of a 150-ns asynchronization time.

We observe that the simultaneous exchange pulse applied to $Q_{2}$ causes a small increase of the exchange coupling within $Q_{3}$ [which is most obvious in Fig. 4(b) by comparing $6 \pi$ rotations]. This crosstalk is taken into account (by tuning individual pulse parameters $\varepsilon_{1,2,3,4}$ ) when demonstrating identical exchange speed for all qubits in Fig. 2.
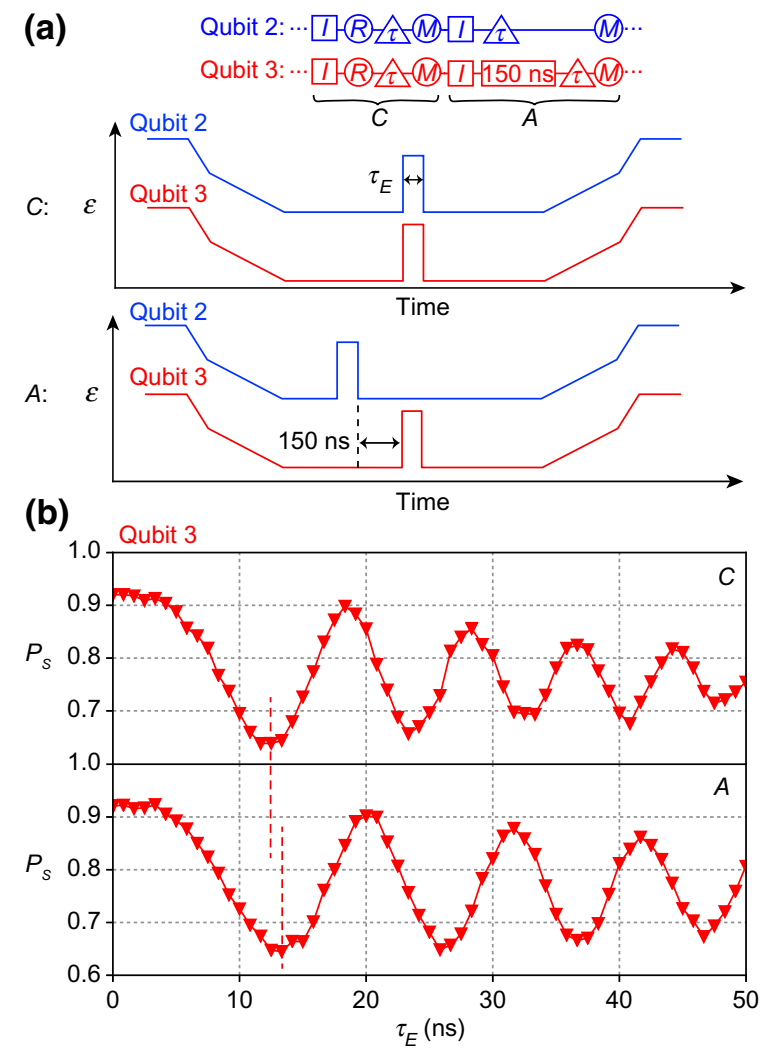

FIG. 4. Crosstalk between qubits. (a) Qubit cycles alternate between protocol $C$ (exchange pulses applied concurrently to $Q_{2}$ and $Q_{3}$ ) and $A$ (pulses applied asynchronously due to a 150 -ns delay). (b) The $Q_{3}$ exchange oscillations in the presence $(C)$ and absence $(A)$ of concurrent exchange pulses applied to $Q_{2}$, indicating that a $\pi$ rotation is obtained $0.9 \mathrm{~ns}$ earlier due to crosstalk from $Q_{2}$ (dashed lines).

\section{E. Coupling to the central multielectron dot}

So far, we have described single-qubit operations. To investigate the possibility of two-qubit operations, we now show coherent spin exchange between qubit $Q_{3}$ and the central multielectron dot (MED). Additional spinexchange data for $Q_{2}$ and $Q_{3}$ are provided in the Appendix.

Previous studies in linear arrays of quantum dots demonstrated coherent spin exchange between two singlettriplet qubits across a MED [18]. The discrete states of the MED played a central role in mediating the spin-spin coupling, but also gave rise to a variety of electron correlation and spin effects - including negative exchange couplings [29] - if only one qubit is coupled with the MED. In that case, we generally expect that a temporary wavefunction overlap between a qubit spin and a MED with odd occupation results in spin dynamics that changes the state of the qubit spin, which can readily be detected as a suppression of $P_{S}$ if the qubit spin was initially part of a singlet pair within the DQD [30]. (Unlike odd occupation, the ground state of an even occupied MED can be spinless, 
and Heisenberg spin exchange with a qubit spin may be absent.)

To search for coherent spin exchange with the MED, we therefore pulse one of the qubits $\left(Q_{3}\right)$ and the MED while keeping the other three DQDs in Coulomb blockade. This allows us to view the pulsed part of the device as a triple quantum dot controlled by $V_{L 3}, V_{R 3}$, and $V_{C}$ with associated stability diagram as sketched in Fig. 5(a). Here, the charge state of this triple dot is indicated by $(m, l, r)$, where $m$ represents the charge state of the multielectron dot while $l$ and $r$ represent the charge states of the left and right wells of the DQD.

After finding a suitable (but unknown) configuration of the multielectron dot ( $m=K$; see the Appendix), we demonstrate coherent exchange coupling with the mediator by repeating the following cycle and determining the fraction of singlet outcomes [refer to Fig. 5(b)]. A singlet pair is prepared in the right well and isolated from the reservoirs by Coulomb blockade $(I)$, by keeping the system within $(K, 0,2)$. The interwell charge transition is then traversed to separate the two entangled electrons from each other $(S)$, by pulsing to $(K, 1,1)$ for the duration of one clock cycle of the waveform generator $(0.83 \mathrm{~ns})$. This operation effectively turns off the intra-DQD exchange interaction while preserving the initialized singlet. Then, an exchange pulse $(E)$ of duration $\tau_{E}$ is applied to $V_{L 3}$, $V_{R 3}$, and $V_{C}$, with an amplitude parameterized by detuning $\varepsilon$ and $\delta$. Here, $\varepsilon=(1 / \sqrt{3})\left(V_{R 3}-V_{R 3}^{o}\right)+(1 / \sqrt{3})\left(V_{L 3}-\right.$ $\left.V_{L 3}^{o}\right)$ and $\delta=(1 / \sqrt{3})\left(V_{C}-V_{C}^{o}\right)$, i.e., for sufficiently large $\varepsilon$ and $\delta$, the triple dot transitions to another charge state, $(K+1,0,1)$, thereby maximizing the wave function overlap between the left qubit spin and the spin of the multielectron dot [Fig. 5(a), inset]. (Here, the $V_{j}^{o}$ are dc operating voltages associated with the three gate electrodes.) The qubit spin is returned to the left well, by pulsing to the separation point in $(K, 1,1)$ for one clock cycle, and

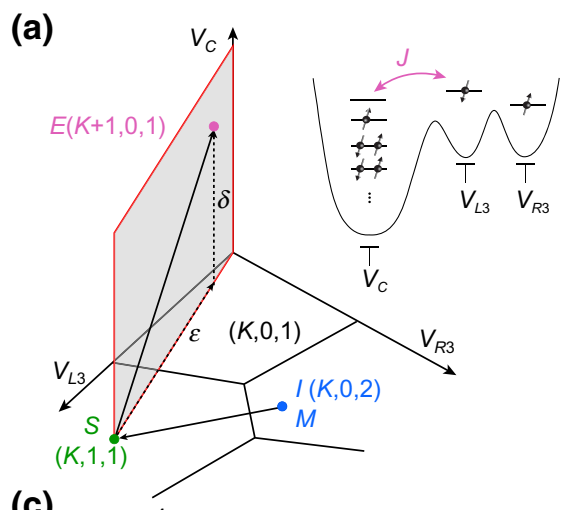

(b)
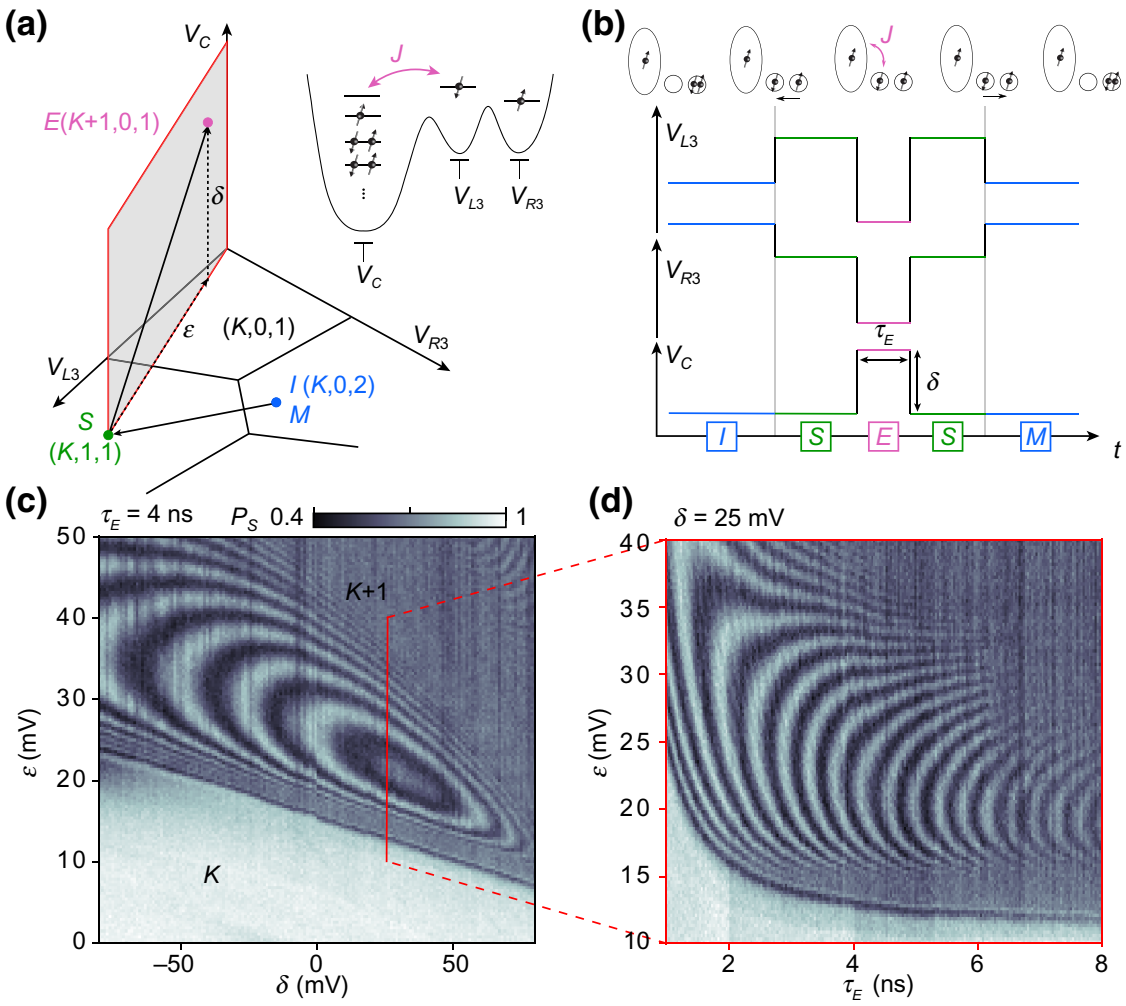

(d)

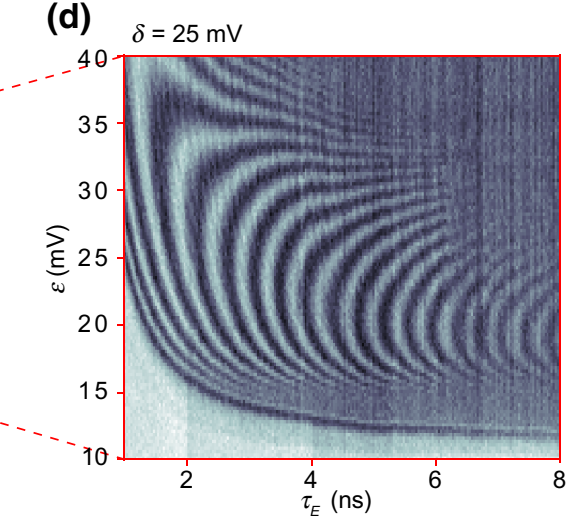

FIG. 5. Coherent spin exchange with the multielectron coupler. (a) Control voltage trajectory that prepares charge state $(K, 1,1)$ with qubit 3 in its spin singlet state $(S)$, before applying simultaneously a detuning pulse $(\varepsilon, \delta)$ for duration $\tau_{E}$. For sufficiently large $\varepsilon$ and $\delta$, spin exchange processes $(E)$ induced by the $(K+1,0,1)$ state are expected to reduce the probability of detecting qubit 3 in a singlet state after pulsing via $S$ and Pauli blockade back to $M$ for measurement. Inset: level structure of the qubit-multielectron system for odd occupation numbers of the coupler, appropriate for results obtained in panels (c) and (d). (b) Waveforms $V_{R 3}(t), V_{L 3}(t)$, and $V_{M}(t)$ used to implement one manipulation cycle. During the exchange pulse $(E), V_{C}$ is pulsed positive relative to $V_{L 3}$ and $V_{R 3}$ to attract the left qubit electron to the multielectron dot. (c) Fraction of singlet outcomes as a function of $\varepsilon$ and $\delta$ for fixed pulse duration. The diagonal threshold for observing oscillations in $P_{S}$ corresponds to the coupler's ground-state transition from odd $(K)$ to even $(K+1)$ occupation number. A large sweet spot emerges near $\delta=30 \mathrm{mV}$ that is further characterized in (d) and Fig. 8. (d) Line cut from (c) for different choices of $\tau_{E}$ and fixed detuning $\delta$. For all pixels in (c) and (d), the cycle in (b) is repeated many times to obtain the fraction of singlet measurements. 
read out by spin-to-charge conversion by pulsing towards $(K, 0,2)$. Any change of the qubit spin during the exchange pulse, coherent or incoherent, is expected to show up as a reduction of $P_{S}$ during the measurement burst $(M)$.

A plot of $P_{S}(\varepsilon, \delta)$ for a fixed exchange pulse duration of 4 ns clearly shows a reduction above a critical pulseamplitude threshold [sloped feature in Fig. 5(c)], which we associate with the charge transition between $(K, 1,1)$ and $(K+1,0,1)$. Above that threshold, $P_{S}$ oscillates as a function of $\varepsilon$ and $\delta$, which we regard as evidence for coherent spin exchange processes from the MED [30].

The ringlike features in $P_{S}\left(\varepsilon, \delta, \tau_{E}=\right.$ constant $)$ can be viewed as contours of constant exchange coupling strength, revealing a nonmonotonic dependence of the exchange coupling on detuning and a prominent maximum. This is also evident if $\tau_{E}$ is varied for fixed $\delta$ [Fig. 5(d)]. A qualitative comparison between this behaviour with a systematic study of a similar multielectron dot [30] suggests that the MED occupation $K$ is odd with an effective ground-state spin of $1 / 2$. In this interpretation, the emergence of a sweet spot and a sign reversal of the exchange coupling strength for larger detuning amplitudes arises from a competition of electron correlations on the multielectron dot and its relatively small level spacing [29].

\section{DISCUSSION}

In this work, using frequency-multiplexed highfrequency reflectometry sensors and eight-dimensional gate-voltage pulses, we demonstrate for the first time the simultaneous coherent manipulation and readout of four spin qubits.

Arranged in a $2 \times 2$ two-dimensional geometry and implemented as singlet-triplet qubits in GaAs, this allows us to sense temporal Overhauser fluctuations at four different sample locations. Our data do not show clear correlations between the four local GaAs nuclear environments. This supports the picture that statistical nuclear polarizations are local [31] and is consistent with nuclear spin diffusion occurring predominantly perpendicular to the plane of the 2DEG, into the heterostructure [32]. However, we did not intentionally induce large nuclear polarizations, as previously done in single-qubit experiments [33]. Our demonstration of spatiotemporal quantum sensing exemplifies the utility of qubit arrays beyond quantum computation, and can be refined in straightforward ways [27].

In addition to simultaneous exchange rotations within the array (with all qubits tuned to the same rotation speed), we also explored the diagnostic value of interleaving different qubit operations. Here, the alternation of Overhauser rotations and exchange rotations revealed potential errors in qubit initialization, providing additional insights for optimizing qubit fidelities that may supplement existing techniques, such as closed-loop control [34,35].
A modification of interleaved operations then allowed us to detect a small change of exchange strengths in the presence of exchange pulses applied to other qubits, which we attribute to a small crosstalk from distant gate electrodes. We expect that automatic compensation of crosstalk will become even more important for controlling larger or denser qubit arrays at high fidelity.

Lastly, we demonstrate coherent spin exchange between one of the qubits and the central multielectron dot. The observed exchange profile is qualitatively in agreement with previous studies in linear arrays [29,30] for an odd-occupied multielectron dot with an effective spin-1/2 ground state. However, additional data (see the Appendix) suggest a prevalence of high-spin ground states that possibly arises from an enhancement of interaction effects by the large size of the multielectron dot, which in future experiments may affect the search for zero-spin ground states suitable for coherent two-qubit coupling [18].

Overall, our results suggest that large quantum dots may prove useful as tunable interqubit couplers to realize twodimensional qubit networks. However, the large number of gate voltages that need to be tuned and synchronized is currently challenging for our manual tune-up procedures, and motivates the development of super-human automation [36].

\section{METHODS}

\section{A. Material and device fabrication}

The device is fabricated on a GaAs/AlGaAs heterostructure with a 2DEG $57 \mathrm{~nm}$ below the surface with carrier density $n=2.5 \times 10^{15} \mathrm{~m}^{-2}$ and mobility $\mu=230 \mathrm{~m}^{2} /$ Vs. The qubit array is realised using standard electron-beam lithography to pattern Ti/Au metallic gates, after the deposition of a 10-nm-thick layer of $\mathrm{HfO}_{2}$. This insulating layer allows the application of both positive and negative gate voltages and obviates the need of bias cooling [22]. For Figs. 1-4 the accumulation gate is held at $+50 \mathrm{mV}$, while it is set to $+75 \mathrm{mV}$ for Fig. 5 .

\section{B. Synchronization of eight-dimensional voltage pulses}

The four qubits are simultaneously controlled by application of eight-dimensional gate-voltage pulses. This required subnanosecond synchronization of two fourchannel arbitrary waveform generators (Tektronix 5014C), which we achieved by triggering both generators with voltage pulses applied via identical cables. In addition, all instruments are synchronized to one $10-\mathrm{MHz}$ frequency standard (SRS FS725).

\section{Four-qubit single-shot readout}

To acquire and analyze the demodulated voltage of all four sensor quantum dots during each waveform cycle, we extend previous approaches for frequency 
multiplexing [24] (see Fig. 6 within the Appendix for details) and state identification [18]. Specifically, during each operation cycle, the four reflectometry tones are temporarily switched on before and after the separation pulse. This allows the acquisition of one reference measurement $\left(V_{\mathrm{RF}}^{R}\right)$ after the singlet initialization, and one actual measurement $\left(V_{\mathrm{RF}}^{M}\right)$ after the qubit operation. To assign single-shot outcomes, the demodulated voltage of each reference burst is subtracted from the demodulated voltage of the measurement burst. The outcomes are then collected into a histogram and fitted with a double Gaussian function. The midpoint between the two Gaussians serves as a voltage threshold, i.e., we assign all outcomes on the singlet (triplet) side to $1(0)$. This identification of qubit states is similar to single-qubit readout in earlier experiments [21], with the additional improvement that the subtraction prior to histogramming makes our method insensitive to small and slow drifts of the sensor operating points, allowing the execution of many qubit cycles over long periods of time.

Similar to previous work [24], we find that the depths of the readout resonances in Fig. 2(b) depend on the tuning (predominantly conductance) of the sensor dots. To improve single-shot readout of the experiments reported in this work, each sensor tuning has been optimized relative to the configuration of Fig. 2(b).

\section{ACKNOWLEDGMENTS}

We thank Fabio Ansaloni for technical help and acknowledge support from the Innovation Fund Denmark and the Independent Research Fund Denmark. A.C. acknowledges support from an EPSRC Doctoral Prize Fellowship. S.F., G.C.G., and M.J.M. grew the heterostructure. F.F. fabricated the device. F.F. and A.C. performed the experiments. F.F., A.C., and F.K. analyzed the data and prepared the manuscript.

\section{APPENDIX: FREQUENCY-MULTIPLEXED READOUT AND ADDITIONAL SPIN EXCHANGE DATA}

\section{Demodulation of four carrier frequencies}

To perform simultaneous qubit readout, we extend to four channels a frequency multiplexing setup similar to that in Ref. [24]. Our setup is shown in Fig. 6 as a circuit schematic and as a photograph of the demodulation circuit.

Four carrier frequencies are generated using independent RF sources (SRS386). Each frequency is passed through a directional coupler that separates the probe tone from the reference tone. Each probe tone is then passed through a voltage-controlled phase shifter, before being added with the other probe tones using a four-port power
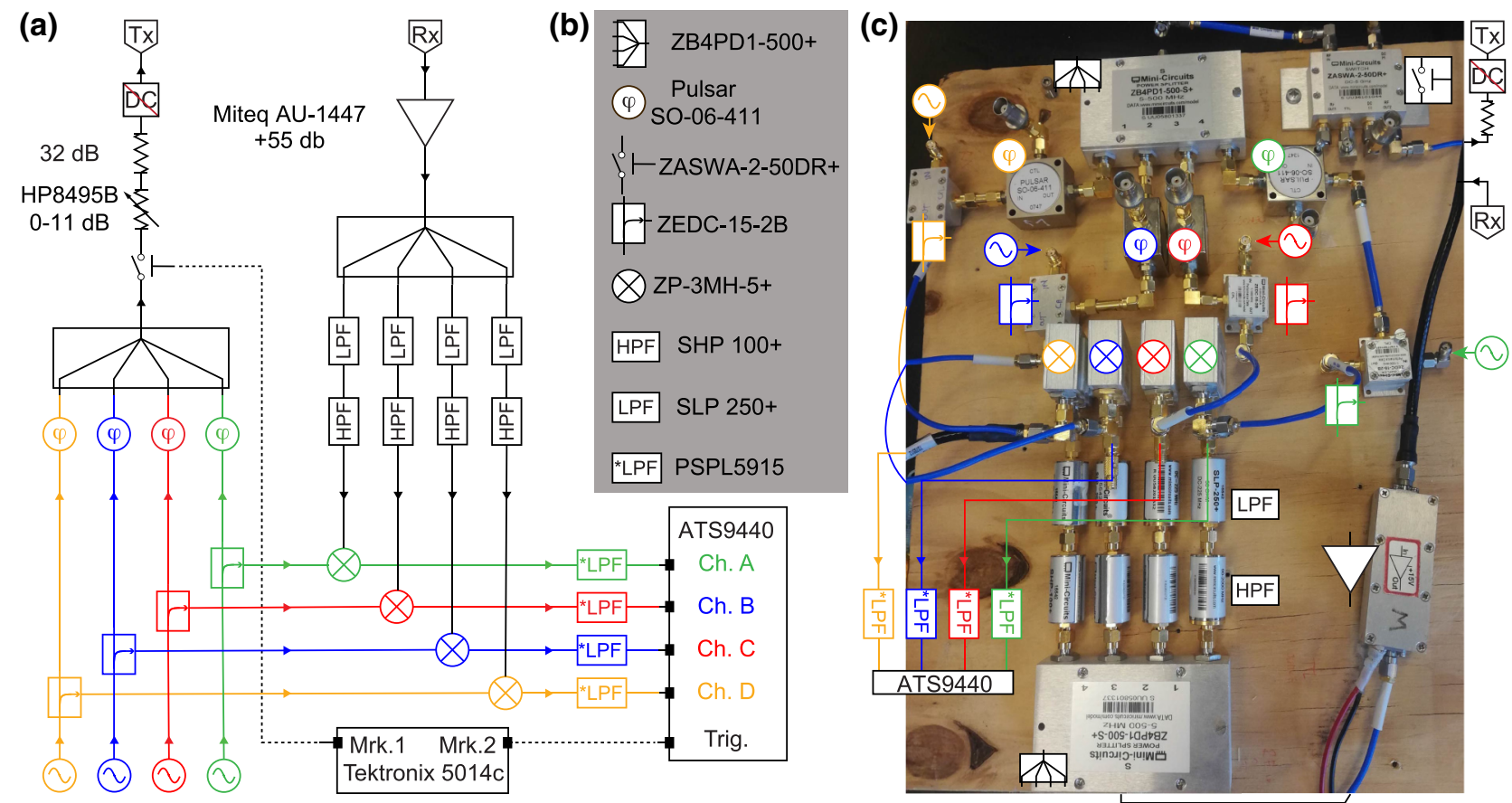

FIG. 6. Four-channel demodulation circuit. (a) Schematic of the circuit layout. Colored lines indicate paths associated with different carrier frequencies, while solid lines indicate paths in which all four rf tones propagate simultaneously. The color code follows that used in the main text. (b) Legend of specific components used in this work. (c) Photograph of the circuit layout. The transmitter and receiver port of the cryostat is denoted by $\mathrm{Tx}$ and $\mathrm{Rx}$, respectively. 
splitter. The resulting signal now contains all four carrier frequencies and is passed through a high-frequency switch, followed by a series of attenuators and filters before being injected into the transmission port (Tx) of the cryostat. Inside the cryostat (not shown), the four carriers are further attenuated before reaching a directional coupler that applies a fraction of the signal to four $L C$ resonators located on the PCB sample holder [23]. Each resonator is wirebonded to an ohmic contact of one of the four sensor quantum dots. Since each resonator is excited by only one of the carrier frequencies (whichever one is resonant with the $L C$ circuit), this provides qubit addressability.

After being reflected by the sample the four carriers are directed by the directional coupler to a cryogenic amplifier before exiting the cryostat at its receiver port (Rx). The signal is then further amplified at room temperature before being split into four equal components by a fourport power splitter. Finally, each component is mixed with one of the reference tones. The demodulated output of each mixer is then low-pass filtered and recorded by a fast digitizer card (Alazar ATS9440).

To implement the reference and measurement bursts described in Sec. IV, we drive the high-frequency switch located before the Tx port with a marker channel of the arbitrary waveform generator [dashed black line in Fig. 6(a)]. A second marker is connected to the trigger port of the Alazar card to mark the beginning of a acquisition. To account for an inevitable delay between the triggers and the instant the signal is received from the demodulation circuit, caused by propagation delays associated with cables and cryostat wiring, we adjust the post-trigger delay of the Alazar card such that its sampling starts after the trigger is received (in our case $500 \mathrm{~ns}$ ).

\section{Additional data for spin exchange with the multielectron dot}

In Fig. 7(a) we show the demodulated voltage measured from $S_{3}$ (after the subtraction of a background plane) as a function of $V_{C}$ and $V_{L 3}$. The resulting charge stability diagram shows horizontal and vertical transitions associated with charging events of the left dot of qubit 3 and the multielectron dot, respectively. White dashed lines highlight interdot charge transitions that can be traversed (along suitable detuning directions $\varepsilon$ ) to test for coherent coupling with the multielectron dot. These data are taken column by column, to allow the multielectron dot sufficient time to exchange electrons with one of the reservoirs while $V_{C}$ is slowly changed, likely via leakage through the $Q_{1}$ or $Q_{4}$ double dot.

In Fig. 7(b) we use the same exchange sequence used for Fig. 5 and measure the demodulated voltage of $S_{3}$ as a function of the detuning parameter $\varepsilon$ and the MED plunger gate $V_{C}$. As function of $V_{C}$, the number of electrons in the MED changes by one approximately every
40-50 mV. Within one charge state, an increase in $V_{C}$ has the same effect as an increase of $\delta$ in Fig. 5(d). We can clearly observe coherent exchange oscillations in several consecutive charge occupations. Since coherent oscillations indicate the presence of an unpaired spin in the MED, this behavior is inconsistent with a single-particle picture of the multielectron dot in which the ground-state spin alternates between $S=0$ and $S=1 / 2$. Deviations from this simple noninteracting picture have previously been observed by Malinowski et al. using a single-triplet qubit coupled to a multielectron dot [30]. We speculate that the ground state of the MED alternates between $S=1 / 2$ and $S=1$ in this region.

The presence of nonzero spin configurations in all three consecutive occupations is further confirmed by leakage spectroscopy measurements; see Fig. 7(c). Leakage spectroscopy data are obtained by pulsing qubit 3 and the MED with the same waveforms used in the main text [see Fig. 5(b)] but with longer interaction time (fixed at $\tau_{E}=150 \mathrm{~ns}$, sufficiently long to detect incoherent spin mixing between the MED and the qubit electron). Markers indicate the choice of $V_{C}$ for each measurement, whereas the triangle indicates the value of $V_{C}$ for the data presented in Figs. 5(c)-5(d).

As a function of detuning $\varepsilon$ and in-plane magnetic field $B_{\|}$, we observe in all three cases a U-like leakage feature. Similar features arise in three-electron triple dots (in Ref. [37] the feature arises from the crossing of two states with different spin projections, denoted as $|\uparrow\rangle S$ and $|\uparrow \uparrow \uparrow\rangle\rangle$ and have also been observed by coupling a singlet-triplet qubit to a spin-1/2 and spin-1 multielectron dot [29,30].

Figure 8 shows the effect of applied magnetic field on the exchange profile of Fig. 5(d). Regarding contours of equal $P_{S}$ as points of constant exchange coupling strength, we observe that in-plane $\left(B_{\|}\right)$and out-of-plane $\left(B_{\perp}\right)$ magnetic fields have no strong effect on the exchange strength. The weak dependence on $B_{\|}$is consistent with similar observations by Martins et. al. for a linear array with somewhat smaller multielectron dot, although they found a stronger dependence on $B_{\perp}$ that was vaguely attributed to orbital magnetic effects [29].

Orbital effects can potentially be studied by changing the size or shape of the multielectron dot by tuning the four large depletion gates ("backbone gates") that are surrounding the elliptical area indicated in Fig. 1(a). We have not systematically studied this capability, but note that, for the experiments in Figs. 5, 7, 8, and 9, the depletion voltages applied to the upper two backbone gates $(-0.70 \mathrm{~V}$ for $Q_{1}$ and $Q_{4}$ ) are more negative relative to the lower two backbone gates $\left(-0.54\right.$ and $-0.56 \mathrm{~V}$ for $Q_{2}$ and $\left.Q_{3}\right)$. In this regime, we do not have direct experimental evidence that the multielectron dot extends all the way underneath the ellipse indicated in Fig. 1(a). (In the regime where tunnel barriers associated with upper and lower qubits are low, and where upper backbone voltages are comparable to 
(a)

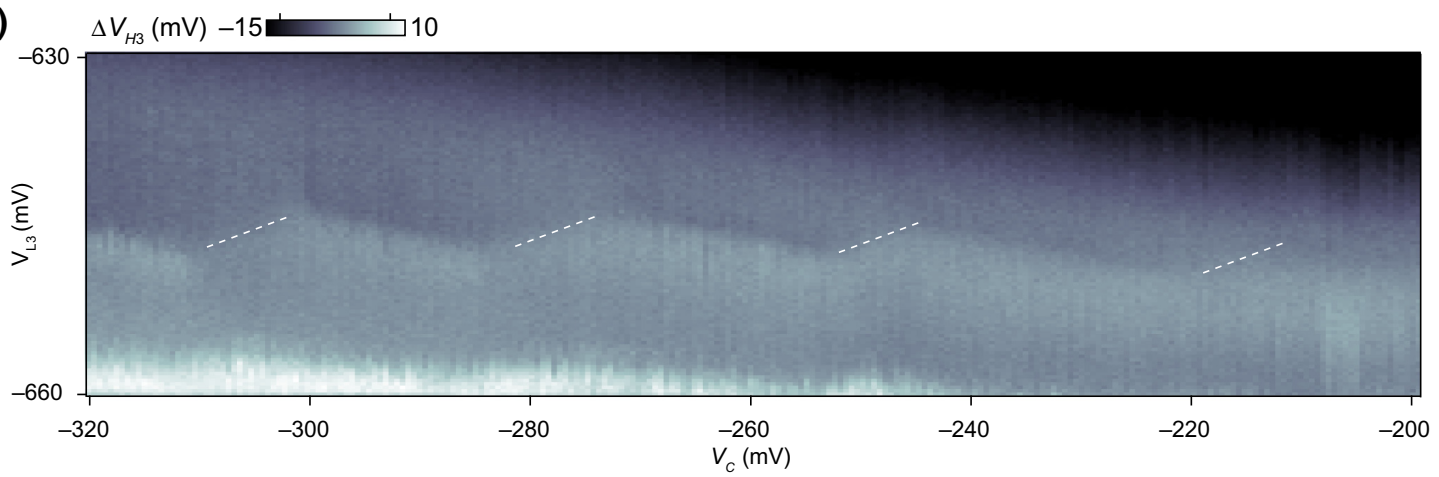

(b)

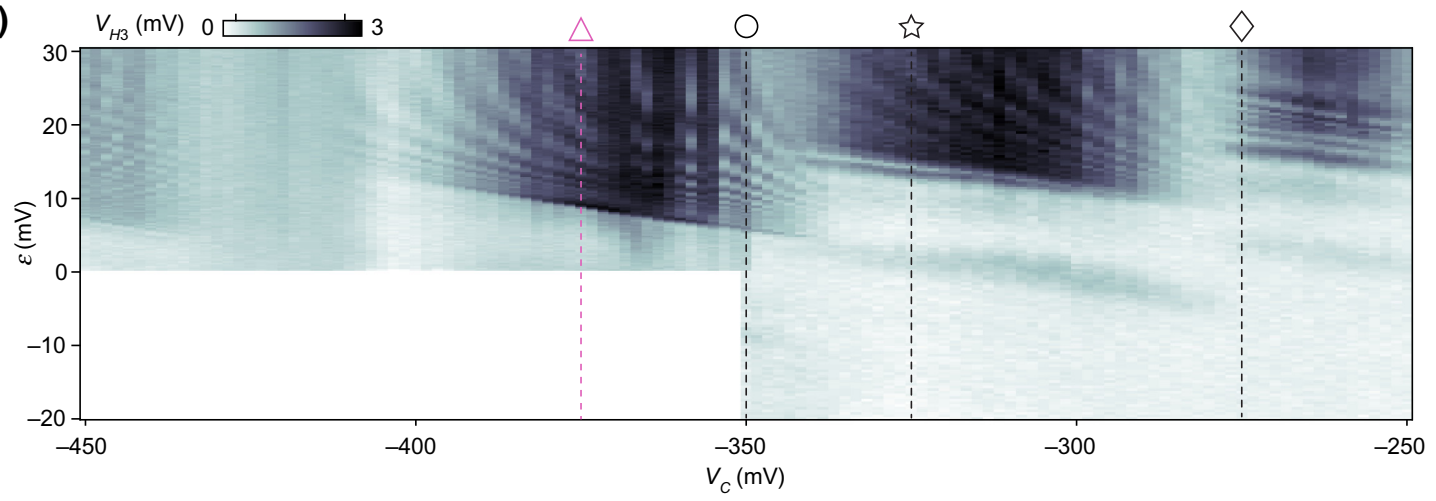

(c)
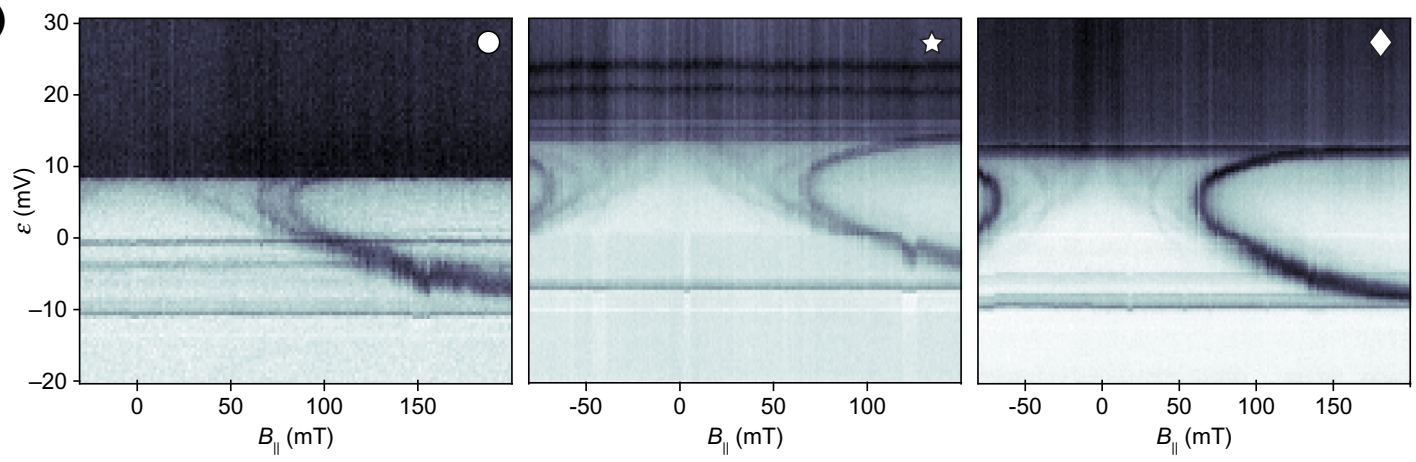

FIG. 7. Coherent spin exchange and leakage spectroscopy of the multielectron dot for several charge occupations. (a) Charge stability diagram of the MED as a function of $V_{C}$ and $V_{L 3}$ in the absence of pulses. Dashed lines indicate interdot transitions between the left dot of qubit 3 and the MED. (b) Demodulated voltage $V_{H 3}$ as a function of detuning parameter $\varepsilon$ and $V_{C}$ for $\tau_{E}=4$ ns, indicating coherent exchange oscillations in several consecutive charge occupations of the MED. The triangular marker indicates $V_{C}$ for the spin exchange presented in Figs. 5, 8, and 9(b). (c) Leakage spectroscopy data [using a fixed interaction time $\tau_{E}=150 \mathrm{~ns}$ and $V_{C}$ values marked in panel (b)] showing $V_{H 3}$ as a function of in-plane magnetic field $B_{\|}$and detuning $\varepsilon$ for three consecutive charge occupations of the multielectron electron dot. In (b) and (c), enhancements of $V_{H 3}$ correspond to a suppression of $P_{S}$, i.e., spin exchange or leakage.

lower backbone voltages, we did verify Coulomb blockade of the fully extended quantum dot by measuring transport between ohmics located on the upper and lower sides of the chip.)

The pulse sequence described in Fig. 5 can also be applied to other qubits. For example, by pulsing $V_{R 2}$ and $V_{L 2}$ instead of $V_{L 3}$ and $V_{R 3}$ we can view the pulsed part of the device as a triple quantum dot comprised of $Q_{2}$ and the multielectron dot [Fig. 9(a)], while $Q_{3}$ remains in Coulomb blockade. The resulting coherent exchange oscillations from $Q_{2}$ (detected by measuring $V_{H_{2}}$ ) are presented as columns in Fig. 9(b) for different voltages applied to the DQD barrier gate $\left(V_{B 2}\right)$. The dependence of exchange oscillations on $V_{B 2}$ suggests that the effective detuning of the multielectron dot and the resulting exchange coupling strength are affected somewhat by capacitive crosscoupling from the barrier gate. During this experiment, all other tuning voltages are identical to those used for the $Q_{3}$ spin-exchange experiments [corresponding to the triangular marker in Fig. 7(b)], indicating that multiqubit coupling to the multielectron dot is possible. However, we have not yet demonstrated qubit-qubit coupling mediated by the multielectron dot (ideally, the coupling of any qubit to any other qubit), but expect that more efficient tuning 

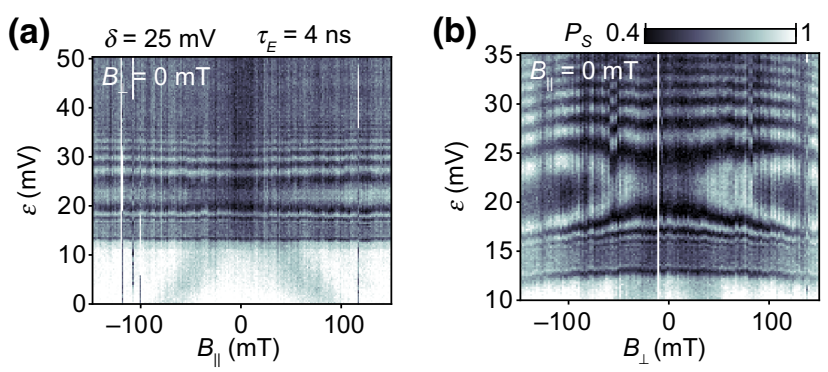

FIG. 8. Dependence of the exchange pattern in Fig. 5 on the applied magnetic field for a fixed amplitude $\delta=25 \mathrm{mV}$ and interaction time $\tau_{E}=4 \mathrm{~ns}$. (a) Fraction of singlet outcomes as a function of $\varepsilon$ and $B_{\|}$for $B_{\perp}=0$. (b) Fraction of singlet outcomes as a function of $\varepsilon$ and $B_{\perp}$ for $B_{\|}=0$.

techniques (for example, based on automation) will allow this in the future.

\section{The $T_{2}^{*}$ fits and exchange quality factors}

Fits for the time averaged data of Fig. 2(c) in the main text are adapted from Ref. [20] using an exponentially damped sine function:

$$
P_{S}(\tau)=C_{1}+C_{2} \sin \left(2 \pi f \tau+C_{3}\right) e^{-\left(\tau / T_{2}^{*}\right)^{2}}
$$

with $C_{1,2,3}, f$, and $T_{2}^{*}$ free parameters. Here, $T_{2}^{*}$ quantifies the inhomogeneous dephasing time due to hyperfine interactions with nuclear spins.

The fits for the exchange oscillations in Fig. 2(d) are obtained using the function

$$
P_{S}(\tau)=C_{1}+C_{2} \cos \left(2 \pi J \tau+C_{3}\right) e^{-\left(\tau / T_{\mathrm{el}}^{*}\right)^{2}}
$$

(a)

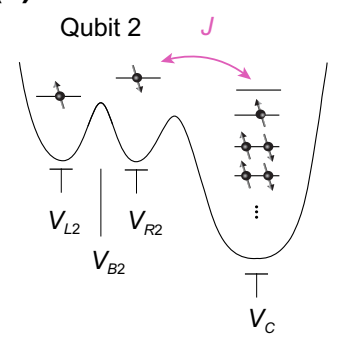

(b)

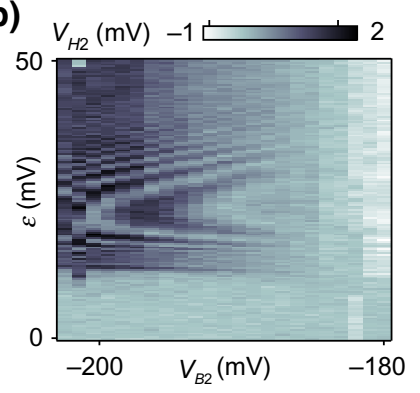

FIG. 9. Coherent spin exchange between $Q_{2}$ and the multielectron dot. (a) Level structure of the qubit-multielectron system similar to that presented for $Q_{3}$ in Fig. 5. Here $V_{B 2}$ is the voltage applied to the interdot barrier gate of $Q_{2}$. (b) Demodulated voltage $V_{H 2}$ as a function of detuning $\varepsilon$ and $V_{B 2}$. For this tuning of sensor 2, an enhanced value of $V_{H 2}$ indicates a suppression of $P_{S}$. with $C_{1,2,3}, J$, and $T_{\mathrm{el}}^{*}$ free parameters. Here, $T_{\mathrm{el}}^{*}$ is sensitive to decoherence arising from noise in the exchange coupling strength $J$, and likely has contributions from effective electrical noise. Quality factors are obtained by $Q=J \times T_{\mathrm{el}}^{*}$ following Ref. [22]. Previous work in Ref. [18] has shown that the high-frequency wiring of the cryostat results in a finite rise time of exchange pulses, which results in deviations from Eq. (A2) for short values of $\tau$. In Fig. 2(d), we therefore exclude data of the first $15 \mathrm{~ns}$ from the fits.

[1] T. F. Watson, B. Weber, Y.-L. Hsueh, L. C. L. Hollenberg, R. Rahman, and M. Y. Simmons, Atomically engineered electron spin lifetimes of $30 \mathrm{~s}$ in silicon, Sci. Adv. 3, e1602811 (2017).

[2] J. T. Muhonen et al., Storing quantum information for 30 seconds in a nanoelectronic device, Nat. Nanotechnol. 9, 986 (2014).

[3] J. Yoneda et al., A quantum-dot spin qubit with coherence limited by charge noise and fidelity higher than $99.9 \%$, Nat. Nanotechnol. 13, 102 (2018).

[4] Y. He, S. K. Gorman, D. Keith, L. Kranz, J. G. Keizer, and M. Y. Simmons, A two-qubit gate between phosphorus donor electrons in silicon, Nature 571, 371 (2019).

[5] T. F. Watson et al., A programmable two-qubit quantum processor in silicon, Nature 555, 633 (2018).

[6] D. M. Zajac, A. J. Sigillito, M. Russ, F. Borjans, J. M. Taylor, G. Burkard, and J. R. Petta, Resonantly driven cnot gate for electron spins, Science 359, 439 (2018).

[7] M. Veldhorst et al., A two-qubit logic gate in silicon, Nature 526, 410 (2015).

[8] A. Chatterjee, P. Stevenson, S. De Franceschi, A. Morello, N. P. de Leon, and F. Kuemmeth, Semiconductor qubits in practice, Nat. Rev. Phys. 3, 157 (2021).

[9] D. M. Zajac, T. M. Hazard, X. Mi, E. Nielsen, and J. R. Petta, Scalable Gate Architecture for a One-Dimensional Array of Semiconductor Spin Qubits, Phys. Rev. Appl. 6, 054013 (2016).

[10] P.-A. Mortemousque, E. Chanrion, B. Jadot, H. Flentje, A. Ludwig, A. D. Wieck, M. Urdampilleta, C. Bäuerle, and T. Meunier, Coherent control of individual electron spins in a two-dimensional quantum dot array, Nat. Nanotechnol. 16, 296 (2021).

[11] Y. Kojima et al., Probabilistic teleportation of a quantum dot spin qubit. arXiv:2011.04881 (2020).

[12] T. Ito et al., Four single-spin rabi oscillations in a quadruple quantum dot, Appl. Phys. Lett. 113, 093102 (2018).

[13] H. Qiao, Y. P. Kandel, K. Deng, S. Fallahi, G. C. Gardner, M. J. Manfra, E. Barnes, and J. M. Nichol, Coherent Multispin Exchange Coupling in a Quantum-Dot Spin Chain, Phys. Rev. X 10, 031006 (2020).

[14] A. Sigillito, J. Loy, D. Zajac, M. Gullans, L. Edge, and J. Petta, Site-Selective Quantum Control in an Isotopically Enriched ${ }^{28} \mathrm{Si} / \mathrm{si}_{0.7} \mathrm{ge}_{0.3}$ Quadruple Quantum dot, Phys. Rev. Appl. 11, 061006 (2019).

[15] K. Takeda, A. Noiri, T. Nakajima, J. Yoneda, T. Kobayashi, and S. Tarucha, Quantum tomography of an entangled three-spin state in silicon. arXiv:2010.10316 (2020). 
[16] N. W. Hendrickx, W. I. Lawrie, M. Russ, F. van Riggelen, S. L. de Snoo, R. N. Schouten, A. Sammak, G. Scappucci, and M. Veldhorst, A four-qubit germanium quantum processor, Nature 591, 580 (2021).

[17] T. Hensgens, T. Fujita, L. Janssen, X. Li, C. Van Diepen, C. Reichl, W. Wegscheider, S. D. Sarma, and L. M. Vandersypen, Quantum simulation of a fermi-hubbard model using a semiconductor quantum dot array, Nature 548, 70 (2017).

[18] F. K. Malinowski et al., Fast spin exchange across a multielectron mediator, Nat. Commun. 10, 1196 (2019).

[19] J. P. Dehollain, U. Mukhopadhyay, V. P. Michal, Y. Wang, B. Wunsch, C. Reichl, W. Wegscheider, M. S. Rudner, E. Demler, and L. M. Vandersypen, Nagaoka ferromagnetism observed in a quantum dot plaquette, Nature 579, 528 (2020).

[20] J. Petta, A. Johnson, J. Taylor, E. Laird, A. Yacoby, M. Lukin, C. Marcus, M. Hanson, and A. Gossard, Coherent manipulation of coupled electron spins in semiconductor quantum dots, Science 309, 2180 (2005).

[21] C. Barthel, M. Kjaergaard, J. Medford, M. Stopa, C. M. Marcus, M. P. Hanson, and A. C. Gossard, Fast sensing of double-dot charge arrangement and spin state with a radiofrequency sensor quantum dot, Phys. Rev. B 81, 161308(R) (2010).

[22] F. Martins, F. K. Malinowski, P. D. Nissen, E. Barnes, S. Fallahi, G. C. Gardner, M. J. Manfra, C. M. Marcus, and F. Kuemmeth, Noise Suppression Using Symmetric Exchange Gates in Spin Qubits, Phys. Rev. Lett. 116, 116801 (2016).

[23] Available from https://www.qdevil.com.

[24] E. A. Laird, J. M. Taylor, D. P. DiVincenzo, C. M. Marcus, M. P. Hanson, and A. C. Gossard, Coherent spin manipulation in an exchange-only qubit, Phys. Rev. B 82, 075403 (2010).

[25] D. Reilly, C. Marcus, M. Hanson, and A. Gossard, Fast single-charge sensing with a rf quantum point contact, Appl. Phys. Lett. 91, 162101 (2007).

[26] F. K. Malinowski, F. Martins, L. Cywiński, M. S. Rudner, P. D. Nissen, S. Fallahi, G. C. Gardner, M. J. Manfra, C. M. Marcus, and F. Kuemmeth, Spectrum of the Nuclear Envi- ronment for Gaas Spin Qubits, Phys. Rev. Lett. 118, 177702 (2017).

[27] P. Szańkowski, M. Trippenbach, and L. Cywiński, Spectroscopy of cross correlations of environmental noises with two qubits, Phys. Rev. A 94, 012109 (2016).

[28] C. Barthel, J. Medford, H. Bluhm, A. Yacoby, C. M. Marcus, M. P. Hanson, and A. C. Gossard, Relaxation and readout visibility of a singlet-triplet qubit in an overhauser field gradient, Phys. Rev. B 85, 035306 (2012).

[29] F. Martins, F. K. Malinowski, P. D. Nissen, S. Fallahi, G. C. Gardner, M. J. Manfra, C. M. Marcus, and F. Kuemmeth, Negative Spin Exchange in a Multielectron Quantum dot, Phys. Rev. Lett. 119, 227701 (2017).

[30] F. K. Malinowski et al., Spin of a Multielectron Quantum dot and its Interaction with a Neighboring Electron, Phys. Rev. X 8, 011045 (2018).

[31] J. Taylor, J. R. Petta, A. C. Johnson, A. Yacoby, C. M. Marcus, and M. D. Lukin, Relaxation, dephasing, and quantum control of electron spins in double quantum dots, Phys. Rev. B 76, 035315 (2007).

[32] J. R. Petta, J. M. Taylor, A. C. Johnson, A. Yacoby, M. D. Lukin, C. M. Marcus, M. P. Hanson, and A. C. Gossard, Dynamic Nuclear Polarization with Single Electron Spins, Phys. Rev. Lett. 100, 067601 (2008).

[33] H. Bluhm, S. Foletti, D. Mahalu, V. Umansky, and A. Yacoby, Enhancing the Coherence of a Spin Qubit by Operating it as a Feedback Loop That Controls its Nuclear Spin Bath, Phys. Rev. Lett. 105, 216803 (2010).

[34] P. Cerfontaine, R. Otten, and H. Bluhm, Self-Consistent Calibration of Quantum-Gate Sets, Phys. Rev. Appl. 13, 044071 (2020).

[35] P. Cerfontaine, T. Botzem, J. Ritzmann, S. S. Humpohl, A. Ludwig, D. Schuh, D. Bougeard, A. D. Wieck, and H. Bluhm, Closed-loop control of a gaas-based singlet-triplet spin qubit with $99.5 \%$ gate fidelity and low leakage”, Nat. Commun. 11, 1 (2020).

[36] T. Moon, Machine learning enables completely automatic tuning of a quantum device faster than human experts, Nat. Commun. 11, 4161 (2020).

[37] J. Medford, J. Beil, J. Taylor, E. Rashba, H. Lu, A. Gossard, and C. M. Marcus, Quantum-Dot-Based Resonant Exchange Qubit, Phys. Rev. Lett. 111, 050501 (2013). 